\title{
ON THE EXISTENCE OF OPTIMAL CONTROLS FOR A CLASS OF NONLINEAR INFINITE DIMENSIONAL SYSTEMS
}

\author{
Elias Flytzanis
}

Athens School of Economics and Business Science, 76 Patission Street, Athens 104-34, Greece

\author{
Nikolaos S. Papageorgiou $\dagger$ \\ Department of Mathematics, University of California, Davis, CA 95616 USA
}

(Submitted by C. Corduneanu)

\begin{abstract}
In this paper we prove the existence of an optimal "state-control" pair for a class of strongly nonlinear systems of subdifferential type with feedback control constraints and an integral cost criterion. Then we establish the existence of optimal solutions for a time optimal control problem involving a moving target set. Finally an example of a distributed parameter system governed by a nonlinear parabolic equation is worked in detail.
\end{abstract}

1. Introduction. In this paper we establish the existence of optimal controls for a large class of strongly nonlinear, distributed parameter systems of monotone type. The problem under consideration is the following:

$$
\left\{\begin{array}{l}
J(x, u)=\int_{0}^{b} L(t, x(t), u(t)) d t \rightarrow \inf \\
\text { s.t. }-\dot{x}(t) \in \partial \psi(x(t))+f(t, x(t)) u(t) \text { a.e. } \\
x(0)=x_{0} \quad u(t) \in U(t, x(t)) \text { a.e., } u(\cdot) \in L^{1}(Y)
\end{array}\right\} .
$$

Our results extend the works of Ahmed [2], Hou [18], Joshi [19], Lions [21] and Vidyasagar [24]. From the above papers, Vidyasagar [24] considers a finite dimensional system and his hypotheses on the data are very strong. Joshi [19] examines a control system governed by a Hammerstein equation. Finally Ahmed [2], Hou [18] and Lions [21] study optimal control problems with dynamics described by a partial differential equation. However in their systems the state and control variables are separated, the control constraints are state independent (open loop) and the overall hypotheses on the data are more restrictive. Recently Ahmed published some very interesting works [3], [4], [5], which are closely related to ours. In those papers Ahmed considers optimal control problems with semilinear dynamics and a Caratheodory cost integrand. However, in some other respects the model considered by Ahmed has some distinct advantages. The forcing term $f(t, x, u)$ is not defined on the whole state space, but only on an appropriate subspace of it, which

Received August 1988, in revised January 1989.

†Research supported by N.S.F. Grant D.M.S. 8802688.

AMS Subject Classifications: 49A27. 
allows for $f(\cdot, \cdot, \cdot)$ to incorporate differential operators that do not have the monotone property, provided they satisfy certain continuity and growth hypotheses (see [4], assumptions (F1)-(F4), pp. 955-956 and [5], p. 41). Furthermore in [3] and [5] Ahmed allows $f(t, x, u)$ to be set valued. Finally in [5] Ahmed also considers stochastic control systems. To our knowledge [5] is the first work on stochastic differential inclusions and their use in optimal control theory. Overall these works of Ahmed contain several new ideas and directions in the study of infinite dimensional control systems, which inspired some generalizations of the results of this work, that will appear in a forthcoming paper (see the remark at the end of the paper). Closing this brief review of the existing literature, we should also mention the recent, important works of Cesari [11], [12], who studied a different class of nonlinear optimal control problems, using results from operator equations and fixed point techniques.

In the next section, we establish our notation and terminology and recall some basic mathematical facts that we will need in the sequel. In Section 3, we prove the existence of optimal controls for system $\left(^{*}\right)$ and we also solve a time optimal control problem for a moving target. Finally in Section 4 we present in detail an example of a control system governed by a nonlinear parabolic partial differential equation, to which our work applies.

2. Mathematical preliminaries. Let $(\Omega, \Sigma)$ be a measurable space and $X$ a separable Banach space. Throughout this paper we will be using the following notation:

$$
P_{f(c)}(X)=\{A \subseteq X: \text { nonempty, closed, (convex) }\}
$$

and

$$
P_{w k c}(X)=\{A \subseteq X: \text { nonempty, weakly compact, convex }\}
$$

A multifunction $F: \Omega \rightarrow P_{f}(X)$ is said to be measurable, if for every $x \in X$, the real valued function $\omega \rightarrow d(x, F(\omega))=\inf \{\|x-y\|: y \in F(\omega)\}$ is measurable. We say that $F: \Omega \rightarrow 2^{X} \backslash\{\emptyset\}$ is graph measurable, if $\operatorname{Gr} F=\{(\omega, x) \in \Omega \times X$ : $x \in F(\omega)\} \in \Sigma \times B(X)$, where $B(X)$ is the Borel $\sigma$-field of $X$. For a $P_{f}(X)$-valued multifunction, measurability implies graph measurability and the converse is true if there exists a complete, $\sigma$-finite measure $\mu(\cdot)$ on $(\Omega, \Sigma)$.

Let $Y, Z$ be Hausdorff topological spaces. A multifunction $G: Y \rightarrow 2^{Z} \backslash\{\emptyset\}$ is said to be upper semicontinuous (u.s.c.), if for all $U \subseteq Z$ nonempty, open, the set $G^{+}(U)=\{y \in Y: G(y) \subseteq U\}$ is open in $Y$.

Now let $H$ be a Hilbert space. By $\Gamma_{0}(H)$ we will denote the space of all $\overline{\mathbf{R}}=$ $\mathbf{R} \cup\{+\infty\}$-valued functions, which are proper (i.e. they are not identically $+\infty$ ), convex and lower semicontinuous (l.s.c.). The subdifferential of $\psi(\cdot)$ at $x$, denoted by $\partial \psi(x)$, is defined by:

$$
\partial \psi(x)=\left\{x^{*} \in X^{*}:\left(x^{*}, y-x\right) \leq \psi(y)-\psi(x) \text { for all } y \in X\right\},
$$

where $(\cdot, \cdot)$ denotes the inner product in $H$. Such elements $x^{*}$ are called the subgradients of $\psi(\cdot)$ at $x$. It is clear from this definition that $\partial \psi: D(\partial \psi) \subseteq H \rightarrow 2^{H}$ is a maximal monotone operator (see Brezis [9]). Also it is easy to see that $\partial \psi(x)$ is always a closed, convex, possibly empty subset of $X$. It is well-known that since $\partial \psi(\cdot)$ 
is a maximal monotone operator, it generates a nonlinear semigroup of contractions $S(t): \overline{D(\partial \psi)} \rightarrow \overline{D(\partial \psi)}, t \geq 0$, via the Crandall-Liggett exponential formula:

$$
S(t) x=\lim _{n \rightarrow \infty}\left(I+\frac{t}{n} \partial \psi\right)^{-n} x
$$

for each $x \in \overline{D(\partial \psi)}$. Furthermore in this case the semigroup has the following smoothing effect: $S(t) \overline{D(\partial \psi)} \subseteq D(\partial \psi)$. We say that the semigroup $\{S(t)\}_{t \geq 0}$ is compact, if for each $t>0$ the operator $S(t)$ is compact. Also $\psi(\cdot) \in \Gamma_{0}(H)$ is said to be of compact type, if for each $\lambda>0$ the level set $L(\lambda)=\left\{x \in H:\|x\|^{2}+\psi(x) \leq \lambda\right\}$ is relatively compact in $H$. From a fundmental theorem of Brezis-Konishi (see [10] and [20]), we know that " $\psi(\cdot)$ is of compact type if and only if the semigroup generated by $-\partial \psi(\cdot)$ on $\overline{D(\partial \psi)}$ is compact." Finally it is known (see Brezis [10]), that a compact semigroup is equicontinuous, i.e. for each bounded set $B \subseteq \overline{D(\partial \psi)}$, the family of functions $\{S(\cdot) x: x \in B\}$ is equicontinuous at each $t>0$.

Closing this section recall that, if $V$ is a separable metrizable space and $U$ a metrizable space, we say that $f: \Omega \times V \rightarrow U$ is a Caratheodory function, if for each $v \in V, \omega \rightarrow f(\omega, v)$ is measurable and for each $\omega \in \Omega, v \rightarrow f(\omega, v)$ is continuous. It is well-known that in this case $(\omega, v) \rightarrow f(\omega, v)$ is jointly measurable.

3. Existence of optimal controls. The mathematical setting is the following. Let $T=[0, b], H$ a separable Hilbert space (the state space) and $Y^{*}$ a separable Banach space ( $Y$ modelling the control space). We will need the following hypotheses on the data of problem $\left({ }^{*}\right)$.

$\mathrm{H}(\psi): \psi \in \Gamma_{0}(H)$ and is of compact type,

$\mathrm{H}(\mathrm{f}): f: T \times H \rightarrow \mathcal{L}(Y, H)$ is a map such that

(1) $t \rightarrow f(t, x) u$ is measurable for each $(x, u) \in H \times Y$,

(2) $(x, u) \rightarrow f(t, x) u$ is continuous from $H \times Y_{W}$ into $H_{W}$ and $x \rightarrow f^{*}(t, x) v$ is continuous from $H$ into $Y^{*}$ for each $v \in Y$ (by $H_{W}$ and $Y_{W}$ we denote the spaces $H$ and $Y$ with their respective weak topologies),

(3) $\|f(t, x)\| \leq a(t)+b(t)\|x\|$ a.e. with $a(\cdot), b(\cdot) \in L_{+}^{2}$.

$\mathrm{H}(\mathrm{U}): U: T \times H \rightarrow P_{f c}(Y)$ is a multifunction such that

(1) $(t, x) \rightarrow U(t, x)$ is graph measurable,

(2) $x \rightarrow U(t, x)$ is u.s.c. from $X$ into $Y_{W}$,

(3) $U(t, x) \subseteq W \in P_{w k c}(Y)$ a.e.,

$\mathrm{H}_{0}: x_{0} \in D(\psi)$,

$\mathrm{H}(\mathrm{L}): L: T \times H \times Y \rightarrow \overline{\mathbf{R}}=\mathbf{R} \cup\{+\infty\}$ is an integrand such that

(1) $(t, x, u) \rightarrow L(t, x, u)$ is measurable,

(2) $(x, u) \rightarrow L(t, x, u)$ is l.s.c. from $H \times Y_{W}$ into $\overline{\mathbf{R}}$ and is convex in $u$,

(3) $h_{1}(t)+h_{2}(t)(\|x\|+\|u\|) \leq L(t, x, u)$ a.e., with $h_{1}, h_{2} \in L^{1}$.

By an admissible "state-control" pair for system $\left(^{*}\right)$, we mean a control function $u(\cdot) \in L^{1}(Y)$ and a state function $x(\cdot) \in C(T, H)$ such that $x(\cdot)$ is a strong solution of the evolution equation describing the dynamics of the system, with $u(\cdot)$ being the driving control function and $u(t) \in U(t, x(t))$ a.e. We will denote the set of admissible "state-control" pairs of $\left(^{*}\right)$ by $P\left(x_{0}\right)$ and by $P_{1}\left(x_{0}\right)$ we will denote the 
corresponding set of trajectories, i.e. $P_{1}\left(x_{0}\right)=\operatorname{proj}_{1} P\left(x_{0}\right) \subseteq C(T, H)$. In order for our problem to be nontrivial, we will need the following "feasibility type" hypothesis:

$\mathrm{H}_{a}: P\left(x_{0}\right) \neq \emptyset$ and there exists $(x, u) \in P\left(x_{0}\right)$ such that $J(x, u)<\infty$.

In the proof of our existence result we will need the following easy lemma, whose proof is straightforward and so is omitted.

Lemma I. If $Z$ is any Banach space, $A \subseteq Z$ is nonempty and for every $\epsilon>0$ there exists $A(\epsilon) \subseteq Z$ relatively compact such that for each $x \in A$ we can find $x(\epsilon) \in A(\epsilon)$ for which $\|x-x(\epsilon)\|<\epsilon$, then $A$ is relatively compact.

Now we are ready to prove the existence theorem for optimal control problem $(*)$. Let $m=\inf \left\{J\left(x^{\prime}, u^{\prime}\right):\left(x^{\prime}, u^{\prime}\right) \in P\left(x_{0}\right)\right\}$ (the value of the problem). Our theorem reads as follows:

Theorem 3.2. If hypotheses $H(\psi), H(f), H(U), H_{0}, H(L)$ and $H_{a}$ hold, then there exists $(x, u) \in P\left(x_{0}\right)$ such that $m=J(x, u)$.

Proof: First let us determine an a priori bound for the elements of $P_{1}\left(x_{0}\right)$. So let $x(\cdot) \in C(T, H)$ be such a trajectory. Denote by $S(t): \overline{D(\partial \psi)} \rightarrow D(\partial \psi)$ the semigroup of nonlinear contractions generated by $-\partial \psi$. From Benilan [8], we know that:

$$
\begin{gathered}
\left\|x(t)-S(t) x_{0}\right\| \leq \int_{0}^{t}\|f(x, x(s)) u(s)\| d s \\
\Longrightarrow\|x(t)\| \leq\left\|S(t) x_{0}\right\|+\int_{0}^{t}(a(s)+b(x)\|x(s)\|) \cdot\|u(s)\| d s \\
\Longrightarrow\|x(t)\| \leq\left\|S(t) x_{0}\right\|+\int_{0}^{t}(a(s)|W|+b(s)|W| \cdot\|x(s)\|) d s,
\end{gathered}
$$

where $|W|=\sup \{\|u\|: u \in W\}$. Recall that $t \rightarrow S(t) x_{0}$ is continuous. So we can find $M>0$ such that $\left\|S(t) x_{0}\right\| \leq M$ for all $t \in T$. Then we can write that:

$$
\|x(t)\| \leq M+\int_{0}^{b} a(s)|W| d s+\int_{0}^{t}|W| \cdot b(s)\|x(s)\| d s .
$$

Applying Gronwall's inequality, we get that:

$$
\|x(t)\| \leq\left(M+|W| \cdot\|a\|_{1}\right) \exp \left(|W| \cdot\|b\|_{1}\right)=M_{1}
$$

for all $t \in T$ and all $x(\cdot) \in P_{1}\left(x_{0}\right)$.

Next let $t^{\prime}, t \in T, t<t^{\prime}$. Given that the elements of $P_{1}\left(x_{0}\right)$ are strong solutions of the evolution equation in $\left(^{*}\right)$, we have:

$x\left(t^{\prime}\right)-x(t)=\int_{t}^{t^{\prime}} \dot{x}(s) d s \Longrightarrow\left\|x\left(t^{\prime}\right)-x(t)\right\| \leq \int_{t}^{t^{\prime}}\|\dot{x}(s)\| d s=\int_{0}^{b}\left\|\chi_{\left[t, t^{\prime}\right]}(s) \dot{x}(s)\right\| d s$.

Applying the Cauchy-Schwartz inequality, we get that:

$$
\left\|x\left(t^{\prime}\right)-x(t)\right\| \leq\left[\int_{0}^{b} \chi_{\left[t, t^{\prime}\right]}^{2}(s) d s\right]^{1 / 2} \cdot\left[\int_{0}^{b}\|\dot{x}(x)\|^{2} d s\right]^{1 / 2} .
$$


But from Theorem 3.6, p. 73 of Brezis [9], we know that:

$$
\begin{aligned}
{\left[\int_{0}^{b}\|\dot{x}(s)\|^{2} d s\right]^{1 / 2} } & \leq\left[\int_{0}^{b}\|f(s, x(s)) u(s)\|^{2} d s\right]^{1 / 2}+\sqrt{\psi\left(x_{0}\right)} \\
& \leq\|\gamma\|^{2}+\sqrt{\psi\left(x_{0}\right)}=M_{2}
\end{aligned}
$$

where $\gamma(s)=\left(a(s)+b(s) M_{1}\right) \cdot|W| \in L_{+}^{2}$. So finally we can write that:

$$
\begin{aligned}
\left\|x\left(t^{\prime}\right)-x(t)\right\| & \leq M_{2}\left(t^{\prime}-t\right)^{1 / 2}, x(\cdot) \in P_{1}\left(x_{0}\right) \Longrightarrow \\
P_{1}\left(x_{0}\right) & \subseteq C(T, H) \text { is equicontinuous. }
\end{aligned}
$$

Now let $t \in(0, b]$ and $\epsilon \in(0, t)$. Consider the following Cauchy problem:

$$
\left\{\begin{array}{l}
\dot{z}(\epsilon)(s) \in \partial \psi(z(\epsilon)(s)) \text { a.e. on }[t-\epsilon, t] \\
z(\epsilon)(t-\epsilon)=x(t-\epsilon), x(\cdot) \in P_{1}\left(x_{0}\right)
\end{array}\right\} .
$$

Once again from Benilan [8], we have that:

$$
\|z(\epsilon)(t)-x(t)\| \leq \int_{t-\epsilon}^{t}\|f(s, x(s)) u(s)\| d s \leq \int_{t-\epsilon}^{t} \gamma(s) d s .
$$

Given $\epsilon^{\prime}>0$, we can pick $\epsilon \in(0, t)$ such that $\int_{t-\epsilon}^{t} \gamma(s) d s<\epsilon^{\prime}$. So we have:

$$
\|z(\epsilon)(t)-x(t)\|<\epsilon^{\prime}
$$

But note that $z(\epsilon)(t)=S(\epsilon) x(t-\epsilon), x(\cdot) \in P_{1}\left(x_{0}\right)$. Since $\psi(\cdot)$ is of compact type, we know that $S(\cdot)$ is a compact semigroup. Hence since $P_{1}\left(x_{0}\right)$ is bounded, we deduce that $\left\{z(\epsilon)(t)=S(\epsilon) x(t-\epsilon): x(\cdot) \in P_{1}\left(x_{0}\right)\right\}$ is relatively compact in $H$. So using Lemma I, we have that $\overline{P_{1}\left(x_{0}\right)(t)}=\overline{\left\{x(t): x(\cdot) \in P_{1}\left(x_{0}\right)\right\}}$ is compact in $H$. Invoking the Arzela-Ascoli theorem, we conclude that $\overline{P_{1}\left(x_{0}\right)}$ is compact in $C(T, H)$.

Next we will show that $P_{1}\left(x_{0}\right)$ is closed in $C(T, H)$. To this end let $x_{n} \rightarrow x$ in $C(T, H), x_{n}(\cdot) \in P_{1}\left(x_{0}\right), n \geq 1$. We have:

$$
-\dot{x}_{n}(t) \in \partial \psi\left(x_{n}(t)\right)+f\left(t, x_{n}(t)\right) u_{n}(t) \text { a.e. }
$$

with $u_{n}(t) \in U\left(t, x_{n}(t)\right)$ a.e.. Set $F(t, x)=f(t, x) U(t, x)$. It is clear from hypotheses $\mathrm{H}(\mathrm{f})$ and $\mathrm{H}(\mathrm{U})$ that $F(t, x) \in P_{w k c}(H)$ for all $(t, x) \in T \times H$. We claim that if $x_{n} \stackrel{s}{\longrightarrow} x$, then $\mathrm{w}-\overline{\lim } F\left(t, x_{n}\right) \subseteq F(t, x)$, where $\mathrm{w}-\overline{\lim } F\left(t, x_{n}\right)=\{z \in H: z=$ $\left.\mathrm{w}-\lim z_{n_{k}}, z_{n_{k}} \in F\left(t, x_{n_{k}}\right), k \geq 1\right\}$. So, by denoting for convenience subsequences as the original sequence, we have for $z \in \mathrm{w}-\varlimsup \lim F\left(t, x_{n}\right), z=\mathrm{w}-\lim z_{n}$ with $z_{n} \in F\left(t, x_{n}\right)$. By definition then $z_{n}=f\left(t, x_{n}\right) u_{n}, u_{n} \in U\left(t, x_{n}\right) \subseteq W$. By passing

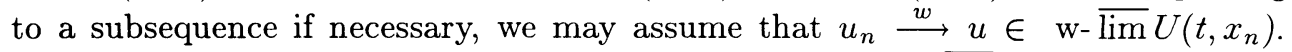
But by hypothesis $U(t, \cdot)$ is u.s.c. from $H$ into $Y_{W}$. So w- $\varlimsup$ im $U\left(t, x_{n}\right) \subseteq U(t, x)$ (see Delahaye-Denel [13]), $\Longrightarrow u \in U(t, x)$. Also from hypothesis $\mathrm{H}(\mathrm{f})(2)$ we have 
that $f\left(t, x_{n}\right) u_{n} \stackrel{w}{\longrightarrow} f(t, x) u$ as $n \rightarrow \infty$. So $z=f(t, x) u$ with $u \in U(t, x) \Longrightarrow z \in$ $F(t, x) \Longrightarrow \mathrm{w}-\varlimsup F\left(t, x_{n}\right) \subseteq F(t, x)$ as claimed.

Set $h_{n}(t)=f\left(t, x_{n}(t)\right) u_{n}(t)$. We know that:

$$
\left\|h_{n}(t)\right\| \leq\left(a(t)+b(t) M_{1}\right) \cdot|W|=\gamma(t) \text { a.e.. }
$$

Invoking the Dunford-Pettis compactness criterion and by passing to a subsequence if necessary, we may assume that $h_{n} \stackrel{w}{\longrightarrow} h$ in $L^{2}(H)$. Then from Theorem 3.1 of [22], we have:

$$
\begin{aligned}
& h(t) \in \overline{\mathrm{conv}} \mathrm{w}-\varlimsup \overline{\lim }\left\{h_{n}(t)\right\}_{n \geq 1} \text { a.e. } \Longrightarrow \\
& h(t) \in \overline{\mathrm{conv}} \mathrm{w}-\varlimsup \overline{\lim } F\left(t, x_{n}(t)\right) \subseteq F(t, x(t)) \text { a.e. }
\end{aligned}
$$

Let $V(t)=\{u \in U(t, x(t)): h(t)=f(t, x(t)) u\}$. Since $h(t) \in F(t, x(t))$ a.e. and from the definition of $F(\cdot, \cdot)$, we see that $V(t) \neq \emptyset$ for all $t \in \bar{T}=T \backslash N, \lambda(N)=0$ ( $\lambda$ being the Lebesgue measure on $T$ ). Then we have:

$$
\operatorname{Gr} V=\{(t, u) \in \bar{T} \times Y: h(t)-f(t, x(t)) u=0\} \cap \operatorname{Gr} U(\cdot, x(\cdot)) .
$$

Note that because of $\mathrm{H}(\mathrm{f})(1)$ and (2), for every $x^{*} \in H,(t, u) \rightarrow\left(x^{*}, h(t)-\right.$ $f(t, x(t)) u)$ is a Caratheodory $\mathbf{R}$-valued function, hence is jointly measurable. So if $\left\{x_{n}^{*}\right\}_{n \geq 1}$ is dense in $H$, we can write that:

$$
\begin{aligned}
& \{(t, u) \in \bar{T} \times Y: h(t)-f(t, x(t)) u=0\} \\
= & \bigcap_{n \geq 1}\left\{(t, u) \in \bar{T} \times Y:\left(x_{n}^{*}, h(t)-f(t, x(t)) u\right)=0\right\} \in B(\bar{T}) \times B(Y) .
\end{aligned}
$$

Also we claim that $t \rightarrow U(t, x(t))=G(t)$ is graph measurable. To see this let $q: T \times Y \rightarrow T \times H \times Y$ be defined by $q(t, y)=(t, x(t), y)$. Clearly $q(\cdot, \cdot)$ is measurable. So the inverse image under $q(\cdot, \cdot)$ of a measurable set is a measurable set. Hence $q^{-1}(\operatorname{Gr} U)=\{(t, y) \in T \times Y:(t, x(t), y) \in \operatorname{Gr} U\}=\{(t, y) \in T \times Y$ : $y \in G(t)=U(t, x(t))\} \in B(T) \times B(Y)$. Therefore we conclude that

$$
\mathrm{Gr} V \in B(\bar{T}) \times B(Y) .
$$

Apply Aumann's selection theorem (see Theorem 5.10 of Wagner [25]), to get $u$ : $T \rightarrow Y$ measurable such that $u(t) \in V(t)$ a.e.. Then we have:

$$
h(t)=f(t, x(t)) u(t) \text { a.e. and } u(t) \in U(t, x(t)) \text { a.e.. }
$$

Also recall that $\left\|\dot{x}_{n}\right\|_{2} \leq\|\gamma\|_{2}+\sqrt{\psi\left(x_{0}\right)}$ for all $n \geq 1$. So once again from the Dunford-Pettis compactness criterion, we may assume that $\dot{x}_{n} \stackrel{w}{\longrightarrow} z$ in $L^{2}(H)$. It is easy to see that $z=\dot{x}$. Note that for $n \geq 1$, we have:

$$
\begin{aligned}
& -\dot{x}_{n}(t)-h_{n}(t) \in \partial \psi\left(x_{n}(t)\right) \text { a.e. } \\
\Longrightarrow & -\dot{x}_{n}(\cdot)-h_{n}(\cdot) \in S_{\partial \psi\left(x_{n}(\cdot)\right)}^{2}=\left\{\text { set of } L^{2}(H) \text {-selectors of } \partial \psi\left(x_{n}(\cdot)\right)\right\} .
\end{aligned}
$$


From Proposition 2.8 of Barbu [7] (see also Theorem 22 of Rockafellar [23]), we know that $S_{\partial \psi\left(x_{n}(\cdot)\right)}^{2}=\partial I_{\psi}\left(x_{n}(\cdot)\right)$, where $I_{\psi}(z(\cdot))=\int_{0}^{b} \psi(z(t)) d t$ if $\psi(x(\cdot)) \in L^{1}$ and $+\infty$ otherwise. The subdifferential $\partial I_{\psi}(\cdot)$ being maximal monotone, has a demiclosed graph, i.e. Gr$\partial I_{\psi}$ is closed in $L^{2}(H) \times L^{2}(H)_{W}$. Observe that $\left(x_{n}(\cdot),-\dot{x}_{n}(\cdot)-\right.$ $\left.h_{n}(\cdot)\right) \in \operatorname{Gr} \partial I_{\psi}, n \geq 1$ and $\left(x_{n}(\cdot),-\dot{x}_{n}(\cdot)-h_{n}(\cdot)\right) \rightarrow(x(\cdot),-\dot{x}(\cdot)-h(\cdot))$ in $L^{2}(H) \times$ $L^{2}(H)_{W} \Longrightarrow(x(\cdot),-\dot{x}(\cdot)-h(\cdot)) \in \mathrm{Gr} \partial I_{\psi} \Longrightarrow-\dot{x}(t)-h(t) \in \partial \psi(x(t))$ a.e. $\Longrightarrow$ $x(\cdot) \in P_{1}\left(x_{0}\right) \Longrightarrow P_{1}\left(x_{0}\right)$ is closed in $C(T, H)$, hence compact.

Now let $\left\{\left(x_{n}, u_{n}\right)\right\}_{n \geq 1}$ be a minimizing sequence of admissible "state-control" pairs, i.e. $\left(x_{n}, u_{n}\right) \in P\left(x_{0}\right), n \geq 1$ and $J\left(x_{n}, u_{n}\right) \downarrow m$. Since $\left\{x_{n}(\cdot)\right\}_{n \geq 1} \subseteq P_{1}\left(x_{0}\right)$ and $\left\{u_{n}(\cdot)\right\}_{n \geq 1} \subseteq S_{W}^{2}=\left\{v \in L^{2}(Y): v(t) \in W\right.$ a.e. $\}$, by passing to a subsequence if necessary, we may assume that $x_{n} \rightarrow x$ in $C(T, H)$ and $u_{n} \stackrel{w}{\longrightarrow} u$ in $L^{2}(Y)$. Then using hypotheses $\mathrm{H}(\mathrm{f})(2)$ and (3), it is easy to see that $f\left(\cdot, x_{n}(\cdot)\right) u_{n}(\cdot) \stackrel{w}{\longrightarrow}$ $f(\cdot, x(\cdot)) u(\cdot)$ in $L^{2}(H)$. Also we may assume that $\dot{x}_{n}(\cdot) \stackrel{w}{\longrightarrow} \dot{x}(\cdot)$ in $L^{2}(H)$. Since $-\dot{x}_{n}(\cdot)-f\left(\cdot, x_{n}(\cdot)\right) u_{n}(\cdot) \in S_{\partial \psi\left(x_{n}(\cdot)\right)}^{2}$, as above we can conclude that $-\dot{x}(\cdot)$ $f(\cdot, x(\cdot)) u(\cdot) \in S_{\partial \psi(x(\cdot))}^{2} \Longrightarrow(x, u) \in P\left(x_{0}\right)$.

Next note that $W$ endowed with the weak topology, is compact, metrizable (see Dunford-Schwartz [5], Theorem 3, p. 434). In the sequel, this will be the topology considered in $W$. From Lemma 2 of Balder [6], we know that we can find $L_{m}: T \times B_{M_{1}} \times W \rightarrow \mathbf{R}$ Caratheodory integrands such that $L_{m} \uparrow L$ as $n \rightarrow \infty$. Here $B_{M_{1}}=\left\{x \in H:\|x\| \leq M_{1}\right\}$. We can choose $L_{m}(\cdot, \cdot, \cdot)$ so that $h(t) \leq$ $L_{m}(t, x, u) \leq m$ a.e. for all $(x, u) \in B_{M_{2}} \times W$, with $h(t)=h_{1}(t)+h_{2}(t)\left(M_{2}+|W|\right)$. Let $M(W)$ denote the Banach space of Borel measures on the compact metric space $W$, with the total variation norm. From the Riesz representation theorem (see Hewitt-Stromberg [17], Theorem 20.48), we know that $[C(W)]^{*}=M(W)$. So from Theorem 1, p. 98 of Diestel-Uhl [14], we have that $\left[L^{1}(T, C(W))\right]^{*}=L^{\infty}(T, M(W))$. Let $\left\{\delta_{u_{n}(\cdot)}(\cdot)\right\}_{n \geq 1} \subseteq L^{\infty}(T, M(W))$, be the Dirac transition measures corresponding to the control functions $\left\{u_{n}(\cdot)\right\}_{n \geq 1}$. Then by Alaoglu's theorem and since the w $^{*}$-topology on $L^{\infty}(T, M(W))$ is metrizable $\left(L^{1}(T, C(W))\right.$ being separable, see Dunford-Schwartz [15], Theorem 1, p. 426), we may assume that $\delta_{u_{n}(\cdot)} \stackrel{w^{*}}{\longrightarrow} \lambda$ in $L^{*}(T, M(W))$. Now note that:

$$
\begin{aligned}
\int_{0}^{b} L_{m}\left(t, x_{n}(t), u_{n}(t)\right) d t & =\int_{0}^{b} \int_{W} L_{m}\left(t, x_{n}(t), z\right) \delta_{u_{n}(t)}(d z) d t \\
& =\int_{0}^{b}\left\langle L_{m}\left(t, x_{n}(t), \cdot\right), \delta_{u_{n}(t)}(\cdot)\right\rangle d t
\end{aligned}
$$

where $\langle\cdot, \cdot\rangle$ denotes the duality brackets for the dual $(C(W), M(W))$. Fix an $m \geq 1$. Then for $n \geq 1$ we have:

$$
\left\|L_{m}\left(t, x_{n}(t), \cdot\right)-L_{m}(t, x(t), \cdot)\right\|_{C(W)}=\sup _{z \in W}\left|L_{m}\left(t, x_{n}(t), z\right)-L_{m}(t, x(t), z)\right| .
$$

Since $L_{m}\left(t, x_{n}(t), \cdot\right)-L_{m}(t, x(t), \cdot)$ is continuous on the compact metric space $W$, we can find $z_{n} \in W$ such that

$$
\left\|L_{m}\left(t, x_{n}(t), \cdot\right)-L_{m}(t, x(t), \cdot)\right\|_{C(W)}=\left|L_{m}\left(t, x_{n}(t), z_{n}\right)-L_{m}\left(t, x(t), z_{n}\right)\right| .
$$


By passing to a subsequence if necessary, we may assume that $z_{n} \stackrel{w}{\longrightarrow} z$. Then exploiting the continuity of $L_{m}(t, \cdot, \cdot)$ on $B_{M_{2}} \times W$, we get that:

$$
\begin{aligned}
& \left|L_{m}\left(t, x_{n}(t), z_{n}\right)-L_{m}\left(t, x(t), z_{n}\right)\right| \rightarrow 0 \quad \text { as } n \rightarrow \infty \\
\Longrightarrow & L_{m}\left(t, x_{n}(t), \cdot\right)=\hat{L}_{m}^{n}(t)(\cdot) \rightarrow L_{m}(t, x(t), \cdot)=\hat{L}_{m}(t)(\cdot) \quad \text { as } n \rightarrow \infty \text { in } C(W) .
\end{aligned}
$$

From the dominated convergence theorem, we then have that:

$$
\hat{L}_{m}^{n}(\cdot) \stackrel{s}{\longrightarrow} \hat{L}_{m} \quad \text { as } n \rightarrow \infty \text { in } L^{1}(T, C(W))
$$

So if we denote by $\langle\cdot, \cdot\rangle_{0}$ the duality brackets for the dual pair $\left(L^{1}(T, C(W)), L^{\infty}(T\right.$, $M(W)))$, we have:

$$
\begin{gathered}
\left\langle\hat{L}_{m}^{n}, \delta_{u_{n}}\right\rangle_{0} \rightarrow\left\langle\hat{L}_{m}, \lambda\right\rangle_{0} \quad \text { as } n \rightarrow \infty \Longrightarrow \\
\int_{0}^{b} L_{m}\left(t, x_{n}(t), u_{n}(t)\right) d t \rightarrow \int_{0}^{b} \int_{W} L_{m}(t, x(t), z) \lambda(t)(d z) d t \quad \text { as } n \rightarrow \infty .
\end{gathered}
$$

On the other hand from the monotone convergence theorem, we have:

$$
\int_{0}^{b} \int_{W} L_{m}(t, x(t), z) \lambda(t)(d z) d t \uparrow \int_{0}^{b} \int_{W} L(t, x(t), z) \lambda(t)(d z) d t \quad \text { as } m \rightarrow \infty .
$$

By a diagonalization process we have:

$$
\int_{0}^{b} L_{m(n)}\left(t, x_{n}(t), u_{n}(t)\right) d t \rightarrow \int_{0}^{b} \int_{W} L(t, x(t), z) \lambda(t)(d z) d t \quad \text { as } n \rightarrow \infty .
$$

Observe that for every $n \geq 1$, we have:

$$
\begin{gathered}
\int_{0}^{b} L_{m(n)}\left(t, x_{n}(t), u_{n}(t)\right) d t \leq \int_{0}^{b} L\left(t, x_{n}(t), u_{n}(t)\right) d t=J\left(x_{n}, u_{n}\right) \Longrightarrow \\
\lim _{n \rightarrow \infty} \int_{0}^{b} L_{m(n)}\left(t, x_{n}(t), u_{n}(t)\right) d t=\int_{0}^{b} \int_{W} L(t, x(t), z) \lambda(t)(d z) d t \\
\leq \lim _{n \rightarrow \infty} J\left(x_{n}, u_{n}\right)=m .
\end{gathered}
$$

Next note that for all $A \in B(T)$ we have:

$$
\int_{A} u_{n}(t) d t \stackrel{w}{\longrightarrow} \int_{A} u(t) d t \quad \text { as } n \rightarrow \infty .
$$

Also since $\delta_{u_{n}(\cdot)}(\cdot) \stackrel{w^{*}}{\longrightarrow} \lambda(\cdot)$ in $L^{\infty}(T, M(W))$, we have:

$$
\int_{A} \int_{W} z \delta_{u_{n}(t)}(d z) d t \rightarrow \int_{A} \int_{W} z \lambda(t)(d z) d t .
$$


Since $\int_{A} u_{n}(t) d t=\int_{A} \int_{W} z \delta_{u_{n}(t)}(d z) d t$, we get that:

$$
\int_{A} u(t) d t=\int_{A} \int_{W} z \lambda(t)(d z) d t \text { for all } A \in B(T) \Longrightarrow u(t)=\int_{W} z \lambda(t)(d z) \text { a.e.. }
$$

Since by hypothesis $\mathrm{H}(\mathrm{L})(3), L(t, x, \cdot)$ is convex, from Jensen's inequality we get:

$$
\begin{aligned}
J(x, u) & =\int_{0}^{b} L(t, x(t), u(t)) d t=\int_{0}^{b} L\left(t, x(t), \int_{W} z \lambda(t)(d z)\right) d t \\
& \leq \int_{0}^{b} \int_{W} L(t, x(t), z) \lambda(t)(d z) d t \leq m .
\end{aligned}
$$

But we saw that $(x, u) \in P\left(x_{0}\right)$. So we have:

$$
J(x, u)=m \Longrightarrow(x, u) \text { is the desired optimal admissible pair. }
$$

We can also solve a time optimal control problem for a moving target. So let $G: T \rightarrow 2^{H} \backslash\{\emptyset\}$ be a time varying target set. We want to reach $G(\cdot)$ in minimum time moving along the trajectories of our system.

To solve this we will need two new hypotheses.

Replacing hypothesis $\mathrm{H}(\mathrm{L})$, will be the following one describing the moving target set.

$\mathrm{H}(\mathrm{G}): G: T \rightarrow P_{f}(H)$ is an u.s.c. multifunction from $T$ into $H_{W}$.

Also we will replace the feasibility hypothesis $\mathrm{H}_{a}$, with the following "controllability type" hypothesis:

$\mathrm{H}_{c}: E=\left\{t \in T: G(t) \cap P_{1}\left(x_{0}\right)(t) \neq \emptyset\right\} \neq \emptyset$, where $P_{1}\left(x_{0}\right)(t)=\{x(t): x(\cdot) \in$ $\left.P_{1}\left(x_{0}\right)\right\}$.

Then our theorem for the time optimal control problem reads as follows:

Theorem 3.2. If hypotheses $H(\psi), H(f), H(U), H_{0}, H(G)$ and $H_{c}$ hold, then there exists time optimal control.

Proof: Let $\tau=\inf E$ and let $t_{n} \downarrow \tau, t_{n} \in E, n \geq 1$. Then by definition we can find $x_{n}(\cdot) \in P_{1}\left(x_{0}\right)$ such that $x_{n}\left(t_{n}\right) \in G\left(t_{n}\right), n \geq 1$. From the proof of Theorem 3.1, we know that $P_{1}\left(x_{0}\right)$ is compact in $C(T, H)$. So we may assume that $x_{n} \rightarrow x \in P_{1}\left(x_{0}\right)$ in $C(T, H)$. Hence $x_{n}\left(t_{n}\right) \stackrel{s}{\longrightarrow} x(\tau)$ as $n \rightarrow \infty$. Since by hypothesis $\mathrm{H}(\mathrm{G}), G(\cdot)$ is u.s.c. into $\mathrm{H}_{W}$, we have that $x(\tau) \in \mathrm{w}-\varlimsup \overline{\lim } G\left(t_{n}\right) \subseteq G(\tau)$. Let $u(\cdot)$ be an admissible control generating $x(\cdot)$, i.e. $(x, u) \in P\left(x_{0}\right)$. Then this is the desired time optimal control.

4. An example. Let $T=[0, b]$ and let $V$ be an open domain in $\mathbf{R}^{n}$ with smooth boundary $\partial V=\Gamma$. On $T \times V$ we consider the following strongly nonlinear, optimal control problem of parabolic type:

$$
J(x, u)=\int_{0}^{b} \int_{V} L(t, z, x(t, z), u(t, z)) d z d t \rightarrow \inf
$$


such that

$$
\begin{aligned}
& \frac{\partial x(t, z)}{\partial t}-\sum_{k=1}^{n} \frac{\partial}{\partial z_{k}}\left[\left|\frac{\partial x(t, z)}{\partial z_{k}}\right|^{p-2} \frac{\partial x(t, z)}{\partial z_{k}}\right] \\
& \quad+|x(t, z)|^{p-2} x(t, z)+f(t, z, x(t, z)) u(t, z)=0 \\
& x(t, z)=0 \quad \text { on } T \times \Gamma \\
& x(0, z)=x_{0}(z) \quad \text { and } \quad \int_{V} u(t, z)^{2} d z \leq \int_{V} k(t, z, x(t, z)) d z, \quad p \geq 2 .
\end{aligned}
$$

We will make the following hypotheses concerning the data of this problem: $\mathrm{H}(\mathrm{f})^{\prime}: f: T \times V \times \mathbf{R} \rightarrow \mathbf{R}$ is a function such that

(1) $(t, z) \rightarrow f(t, z, x)$ is measurable,

(2) $x \rightarrow f(t, z, x)$ is continuous,

(3) $|f(t, z, x)| \leq a(t, z)$ a.e. with $a(\cdot, \cdot)$ measurable, $a(t, \cdot) \in L^{\infty}(V)$ and

$$
t \rightarrow\|a(t, \cdot)\|_{L^{\infty}(V)} \in L^{2}(T),
$$

$\mathrm{H}(\mathrm{k}): k: T \times V \times \mathbf{R} \rightarrow \mathbf{R}$ is a function such that

(1) $(t, z) \rightarrow k(t, z, x)$ is measurable,

(2) $x \rightarrow k(t, z, x)$ is continuous,

(3) $k(t, z, x) \leq \mu(z)$ a.e. with $\mu(\cdot) \in L_{+}^{1}$,

$\mathrm{H}(\mathrm{L}): L: T \times V \times \mathbf{R} \times \mathbf{R} \rightarrow \overline{\mathbf{R}}=\mathbf{R} \cup\{+\infty\}$ is an integrand such that

(1) $(t, z, x, u) \rightarrow L(t, z, x, u)$ is measurable,

(2) $(x, u) \rightarrow L(t, z, x, u)$ is l.s.c. and convex in $u$,

(3) $h(t, z) \leq L(t, z, x, u)$ a.e. with $h(\cdot, \cdot) \in L^{1}(T \times V)$,

$\mathrm{H}_{0}^{\prime}: x_{0}(\cdot) \in W_{0}^{1, p}(V)$,

$\mathrm{H}_{a}^{\prime}$ : there exists an admissible "state-control" pair $(x(\cdot, \cdot), u(\cdot, \cdot))$ such that $J(x, u)<\infty$.

We will put problem $(* *)$ in the form of $(*)$. To this end let:

$$
R_{p}(y)=\sum_{k=1}^{n} \frac{\partial}{\partial z_{k}}\left[\left|\frac{\partial y}{\partial z_{k}}\right|^{p-2} \frac{\partial y}{\partial z_{k}}\right]-|y|^{p-2} y
$$

with domain $D\left(R_{p}\right)=\left\{y \in W_{0}^{1, p}(V): R_{p}(y) \in L^{2}(V)\right\}$.

Also define $\psi: L^{2}(V) \rightarrow \overline{\mathbf{R}}=\mathbf{R} \cup\{+\infty\}$ by:

$$
\psi(y)= \begin{cases}\frac{1}{p} \sum_{k=1}^{n} \int_{V}\left|\frac{\partial y}{\partial z_{k}}\right|^{p} d z+\frac{1}{p} \int_{V}|y(z)|^{p} d z & \text { if } y \in W_{0}^{1, p}(V) \\ +\infty & \text { otherwise }\end{cases}
$$

From Barbu [7], we know that $\psi \in \Gamma_{0}\left(L^{2}(V)\right)$ and that $-R_{p}(y)=\partial \psi(y)$.

The next lemma shows that $\psi(\cdot)$ satisfies hypothesis $\mathrm{H}(\psi)$. 
Lemma II. If $\psi: L^{2}(V) \rightarrow \overline{\mathbf{R}}$ is defined as above, then $\psi(\cdot)$ is of compact type.

Proof: Let $\lambda \geq 0$ and consider the level set $L(\lambda)=\left\{y \in L^{2}(V):\|y\|_{2}^{2}+\psi(y) \leq \lambda\right\}$. Clearly this is an $L^{2}(V)$-bounded set. Also note that since $p \geq 2$, the set $\{|\nabla y|$ : $y \in L(\lambda)\}$ is $L^{2}(V)$-bounded too. Therefore $L(\lambda)$ is bounded in $H^{1}(V)$. But by the Sobolev embedding theorem (see Adams [1], p. 97), we know that $H^{1}(V) \hookrightarrow L^{2}(V)$ compactly. So $L(\lambda))$ is compact in $L^{2}(V)$.

Next let $\hat{f}: T \times L^{2}(V) \rightarrow \mathcal{L}\left(L^{2}(V)\right)$ be defined by

$$
(\hat{f}(t, x) u)(z)=f(t, z, x(z)) u(z), \quad u \in L^{2}(V) .
$$

First let us check that indeed $(\hat{f}(t, x) u)(\cdot) \in L^{2}(V)$. We have:

$$
\begin{aligned}
\int_{V}|(\hat{f}(t, x) u)(z)|^{2} d z & =\int_{V}|f(t, z, x(z)) u(z)|^{2} d z=\int_{V}|f(t, z, x(z))|^{2} \cdot|u(z)|^{2} d z \\
& \leq \int_{V} a(t, z)^{2}|u(z)|^{2} d z \leq\|a(t, \cdot)\|_{L^{\infty}(V)}^{2} \cdot\|u\|_{L^{2}(V)}^{2} \\
\Longrightarrow\|\hat{f}(t, x)\|_{L^{2}(V)} & \leq\|a(t, \cdot)\|_{L^{\infty}(V)}<\infty .
\end{aligned}
$$

So indeed $\hat{f}(t, x)(\cdot) \in \mathcal{L}\left(L^{2}(V)\right)$. Next let $h(\cdot) \in L^{2}(V)$. Then we have:

$$
(h, \hat{f}(t, x) u)_{L^{2}(V)}=\int_{V} h(z)(\hat{f}(t, x) u)(z) d z=\int_{V} h(z) f(t, z, x(z)) u(z) d z .
$$

From Fubini's theorem we know that $t \rightarrow \int_{V} h(v) f(t, z, x(z)) u(z) d z$ is measurable. Since $h(\cdot) \in L^{2}(V)$ was arbitrary, we deduce that $t \rightarrow \hat{f}(t, x) u$ is weakly measurable from $T$ into $L^{2}(V)$. But $L^{2}(V)$ is separable. So from Pettis measurability theorem (see Diestel-Uhl[14], Theorem 2, p. 42), we conclude that $t \rightarrow \hat{f}(t, x) u$ is measurable from $T$ into $L^{2}(V)$.

Next $\left(x_{n}, u_{n}\right) \rightarrow(x, u)$ in $L^{2}(V) \times L^{2}(V)_{W}$. By passing to a subsequence if necessary, we may assume that $x_{n}(z) \rightarrow x(z)$ a.e.. Then for every $h(\cdot) \in L^{2}(V)$ we can easily see that $\int_{V} h(z) f\left(t, z, x_{n}(z)\right) u_{n}(z) d z \rightarrow \int_{V} h(z) f(t, z, x(z)) u(z) d z \Longrightarrow$ $(x, u) \rightarrow \hat{f}(t, x) u$ is continuous from $L^{2}(V) \times L^{2}(V)_{W}$ into $L^{2}(V)_{W}$. Also note that:

$$
\begin{aligned}
(\hat{f}(t, x) u, h)_{L^{2}(V)} & =\int_{V} f(t, z, x(z)) u(z) h(z) d z=\int_{V} u(z) f(t, z, x(z)) h(z) d z \\
& =\left(u, \hat{f}^{*}(t, x) h\right)_{L^{2}(V)} \Longrightarrow \hat{f}(t, x)=\hat{f}^{*}(t, x) .
\end{aligned}
$$

Finally from an earlier calculation, we have that:

$$
\|\hat{f}(t, x) u\|_{L^{2}(V)}^{2} \leq\|a(t, \cdot)\|_{L^{\infty}(V)}^{2} \cdot\|u\|_{L^{2}(V)}^{2} \Longrightarrow\|\hat{f}(t, x)\|_{\mathcal{L}\left(L^{2}(V)\right)} \leq \hat{a}(t)
$$

where $\hat{a}(t)=\|a(t, \cdot)\|_{L^{\infty}(V)} \in L_{+}^{2}$ (see hypothesis $\left.\mathrm{H}(\mathrm{f})^{\prime}(3)\right)$. So we have checked that $\hat{f}(t, x) u$ satisfies hypothesis $\mathrm{H}(\mathrm{f})$.

Next define $\hat{L}: T \times L^{2}(V) \times L^{2}(V) \rightarrow \overline{\mathbf{R}}=R \cup\{+\infty\}$ by:

$$
\hat{L}(t, x, u)=\int_{V} L(t, z, x(z)) d z .
$$


Again from Lemma 2 of Balder [6], we can find $L_{m}: T \times V \times \mathbf{R} \times \mathbf{R} \rightarrow \mathbf{R}$ Caratheodory functions such that $h(t, z) \leq L_{m}(t, z, x, u) \leq m$ and $L_{m} \uparrow L$ as $m \rightarrow \infty$. Set

$$
\hat{L}_{m}(t, x, u)=\int_{V} L_{m}(t, z, x(z), u(z)) d z .
$$

From Fubini's theorem, $t \rightarrow \hat{L}_{m}(t, x, u)$ is measurable, while it is clear that $(x, u) \rightarrow$ $\hat{L}_{m}(t, x, u)$ is continuous. So $\hat{L}_{m}(t, x, u)$ is a Caratheodory map, hence jointly measurable. Applying the monotone convergence theorem, we have that:

$$
\hat{L}_{m}(t, x, u) \uparrow \hat{L}(t, x, u) \Longrightarrow(t, x, u) \rightarrow \hat{L}(t, x, u) \text { is measurable. }
$$

Also because of hypothesis $\mathrm{H}(\mathrm{L})^{\prime}(2)$, we have that $\hat{L}(t, x, \cdot)$ is convex, while from Theorem 2.1, p. 243 of Ekeland-Temam [16] and hypothesis $\mathrm{H}(\mathrm{L})^{\prime}$, we have that $(x, u) \rightarrow \hat{L}(t, x, u)$ is 1.s.c. from $L^{2}(V) \times L^{2}(V)_{W}$ into $\overline{\mathbf{R}}=\mathbf{R} \cup\{+\infty\}$. Finally if we set $\hat{\psi}(t)=\int_{V} \psi(t, z) d z$, then $\hat{\psi}(\cdot) \in L^{1}$ and $\hat{\psi}(t) \leq \hat{L}(t, x, u)$ a.e. for all $(x, u) \in L^{2}(V) \times L^{2}(V)$. So we see that $\hat{L}(t, x, u)$ satisfies hypothesis $\mathrm{H}(\mathrm{L})$.

Next let $U: T \times L^{2}(V) \rightarrow P_{f c}\left(L^{2}(V)\right)$ be defined by:

$$
U(t, x)=\left\{u \in L^{2}(V):\|u\|_{L^{2}(V)}^{2} \leq \hat{k}(t, x)\right\}
$$

where $\hat{k}(t, x)=\int_{V} k(t, z, x(z)) d z$. Clearly then $\hat{k}(t, x)$ is measurable in $t$ and continuous in $x$ (see hypothesis $\mathrm{H}(\mathrm{k}))$. Set $q(t, x, u)=\|u\|_{L^{2}(V)}^{2}-\hat{k}(t, x)$. This function is measurable in $t$ and continuous in $(x, u)$. Thus it is jointly measurable. Therefore we have that:

$$
\begin{aligned}
\operatorname{Gr} U & =\left\{(t, x, u) \in T \times L^{2}(V) \times L^{2}(V): q(t, x, u)=0\right\} \\
& \in B(T) \times B\left(L^{2}(V)\right) \times B\left(L^{2}(V)\right) \Longrightarrow U(\cdot, \cdot) \text { is graph measurable. }
\end{aligned}
$$

Also from hypothesis $\mathrm{H}(\mathrm{k})$ (3) we see that $|U(t, x)| \leq \sqrt{\|\mu\|}_{L^{1}(V)}=M$. So if we set $B_{M}(0)=$ closed ball of radius $M$, centered at 0$\}=W$, then we have $W \in$ $P_{w k c}\left(L^{2}(V)\right)$. So in order to check that $x \rightarrow U(t, x)$ is u.s.c. from $L^{2}(V)$ into $L^{2}(V)_{W}$, it suffices to show that $\operatorname{Gr} U(t, \cdot)$ is closed in $L^{2}(V) \times L^{2}(V)_{W}$. To this end let $\left(x_{n}, u_{n}\right) \rightarrow(x, u)$ in $L^{2}(V) \times L^{2}(V)_{W}$, with $\left(x_{n}, u_{n}\right) \in \operatorname{Gr} U(t, \cdot)$. Then we have:

$$
\begin{aligned}
\left\|u_{n}\right\|_{L^{2}(V)}^{2} & \leq \hat{k}\left(t, x_{n}\right) \Longrightarrow\|u\|_{L^{2}(V)}^{2} \leq \underline{\lim \left\|u_{n}\right\|_{L^{2}(V)}^{2}} \\
& \leq \lim \hat{k}\left(t, x_{n}\right)=\hat{k}(t, x) \\
& \Longrightarrow u \in U(t, x) \text {, i.e. } U(t, \cdot) \text { is u.s.c. from } L^{2}(V) \text { into } L^{2}(V)_{W} .
\end{aligned}
$$

So we have seen that $U(t, x)$ satisfies hypothesis $\mathrm{H}(\mathrm{U})$.

Finally set $\hat{x}_{0}=x_{0}(\cdot) \in W_{0}^{1, p}(V)=D(\psi)$ and so hypothesis $\mathrm{H}_{0}$ is satisfied, while hypothesis $\mathrm{H}_{a}$ is satisfied because of $\mathrm{H}_{a}^{\prime}$.

Now let $H=L^{2}(V), Y=L^{2}(V)$ and write problem (**) as the following abstract control problem:

$$
\left\{\begin{array}{c}
\hat{J}(x, u)=\int_{0}^{b} \hat{L}(t, x(t), u(t)) d t \rightarrow \inf \\
\text { s.t. }-\dot{x}(t) \in \partial \psi(x(t))+\hat{f}(t, x(t)) u(t) \text { a.e. } \\
x(0)=x_{0} \quad u(t) \in U(t, x(t)) \text { a.e. }
\end{array}\right\}
$$


Note that problem $\left({ }^{* * \prime}\right)$ falls into the class of abstract optimization problems studied in Section 3. So we can state the following existence theorem concerning problem $(* *)$.

Theorem 4.1. If hypotheses $H(f)^{\prime}, H(\mathrm{k}), H(L)^{\prime}, H_{0}^{\prime}$ and $H_{a}^{\prime}$ hold, then there exist $u(\cdot, \cdot) \in L^{2}(T \times V)$ and $x(\cdot) \in C\left(T, L^{2}(V)\right)$ such that $\dot{x}(\cdot) \in L^{2}\left(T, L^{2}(V)\right), x(\cdot) \in$ $L^{\infty}\left(T, W_{0}^{1, p}(V)\right)$ and $J(x, u)=m$.

Proof: From the work done above, we see that the equivalent abstract problem $\left({ }^{* * \prime}\right)$ satisfies all the hypotheses of Theorem 3.1. So the result follows from Theorem 3.6 of Brezis [9] (p. 72) and Theorem 3.1 of this paper.

Remark. We can allow the control to enter nonlinearly in the dynamics of the system and drop the convexity hypothesis on $u \rightarrow L(t, x, u)$ and instead assume that for every $(t, x) \in T \times H$, the set $Q(t, x)=\{(\eta, v): \eta \geq L(t, x, u), v \in$ $\partial \psi(x)+f(t, x, u), u \in U(t, x)\}$ is convex. Then using the "reduction technique" of Cesari-Rockafellar, we can still establish the existence of optimal admissible pairs for the control system (see also Ahmed [3], [5]). The details of this extension will appear in a forthcoming paper.

Acknowledgement. The authors would like to express their gratitude to the referee for bringing to their attention the very interesting work of Ahmed and for other suggestions and remarks that helped improve the content of this paper.

\section{REFERENCES}

[1] R. Adams, "Sobolev Spaces," Academic Press, New York, 1975.

[2] N.U. Ahmed, Optimal control of a class of strongly nonlinear parabolic systems, J. Math. Anal. Appl. 61 (1977), 188-207.

[3] N.U. Ahmed, Existence of optimal controls for a class of systems governed by differential inclusions on a Banach space, J. Optim. Theory and Appl. 50 (1986), 213-237.

[4] N.U. Ahmed, Properties of relaxed trajectories for a class of nonlinear evolution equations on a Banach space, SIAM J. Control Optim. 21 (1983), 953-967.

[5] N.U. Ahmed, Existence of optimal relaxed controls for differential inclusions on Banach space, in "Nonlinear Analysis and Applications," ed. V. Lakshmikantham, Proc. of the 7th International Conference on Nonlinear Analysis and Applications, Lecture Notes in Pure and Appl. Math. Vol. 109, Marcel Dekker Inc., 1987, 39-49.

[6] E. Balder, Lower semicontinuity of integral functionals with nonconvex integrands by relaxation-compactification, SIAM Jour. Control Optim. 19 (1981), 533-541.

[7] V. Barbu, "Nonlinear Semigroups and Differential Equations in Banach spaces," Noordhoff Intern. Publishing, Leyden, The Netherlands, 1976.

[8] Ph. Benilan, Solutions integrales d'equations d'evolution dans un espace de Banach, C.R. Acad. Sci. Paris 274 (1972), 47-50.

[9] H. Brezis, "Operateurs Maximaux Monotones et Semigroupes de Contractions dans un Espace de Hilbert," North Holland, Amsterdam, 1973.

[10] H. Brezis, New results concerning monotone operators and nonlinear semigroups, Proceedings of the R.I.M.S. on "Analysis of Nonlinear Problems," Kyoto University (1975), 2-27.

[11] L. Cesari, Existence of solutions and existence of optimal solutions, in "Mathematical Theories of Optimization," eds. M. Cecconi and T. Zolezzi, Lecture Notes in Math. 979, Springer, Berlin, 1983, 88-107.

[12] L. Cesari, Existence of solutions of nonlinear differential equations via fixed point theorems, J. Math. Phys. Sci. 18 (1984), 23-41.

[13] J.-P. Delahaye and J. Denel, The continuities of the point to set maps definitions and equivalences, Math. Progr. Study 10 (1979), 8-12.

[14] J. Diestel and J.J. Uhl, "Vector Measures," Math. Surveys 15, Amer. Math. Soc., Providence, Rhode Island, 1977. 
[15] N. Dunford and J. Schwartz, "Linear Operators I," Wiley, New York, 1958.

[16] I. Ekeland and R. Temam, "Convex Analysis and Variational Problems," North Holland, Amsterdam, 1974.

[17] E. Hewitt and K. Stromberg, "Real and Abstract Analysis," Springer, New York, 1965.

[18] S.-H. Hou, Existence theorems of optimal control problems in Banach spaces, Nonl. Anal. T.M.A. 7 (1983), 239-257.

[19] M. Joshi, On the existence of optimal controls in Banach spaces, Bull. Austr. Math. Soc. 27 (1983), 395-401.

[20] Y. Konishi, Compacité des resolvantes des operateurs maximaux cycliquement monotones, Proc. Japan. Acad. 49 (1973), 303-305.

[21] J.-L. Lions, Optimisation pour certaines classes d'equations d'evolution nonlineaires, Ann. Mat. Pura et Appl. 72 (1966), 275-294.

[22] N.S. Papageorgiou, Convergence theorems for Banach space valued integrable multifunctions, Intern. J. Math. and Math. Sci. 10 (1987), 433-442.

[23] R.T. Rockafellar, "Conjugate duality and optimization," Conf. Board of Math. Sci. Series, No. 16, SIAM Publications, Philadelphia, 1974.

[24] M. Vidyasagar, On the existence of optimal controls, J. Optim. Theory Appl. 17 (1975), 273-278.

[25] D. Wagner, Survey of measurable selection theorems, SIAM J. Control and Optim. 15 (1977), 859-903. 